\title{
A Real-Time System for Facial Expression Recognition using Support Vector Machines and $\boldsymbol{k}$-Nearest Neighbor Classifier
}

\author{
Hend Ab. ELLaban \\ Computer Inst. Prep. Dep. \\ Damietta University \\ Egypt
}

\author{
A. A. Ewees \\ Computer Inst. Prep. Dep. \\ Damietta University \\ Egypt
}

\author{
Elsaeed E. AbdElrazek \\ Computer Inst. Prep. Dep. \\ Damietta University \\ Egypt
}

\begin{abstract}
Faces are a unique feature of human being that can detect a great deal of information about age, health, personalities and feelings. Facial Expressions are the main sources in determining the internal impressions of the individual. RealTime system for facial expression recognition is able to detect and locate human faces in image sequences obtained in real environments then extracts expression features from these images finally recognize facial expressions. In this paper, the proposed system presents a real-time system for facial expression recognition that aims to recognize 8 basic facial expressions of students: anger, disgust, fear, happy, nervous, sad, surprise and natural inside E-learning environment. The primary objective is to use $k-\mathrm{NN}$ and SVM classifiers to test the efficiency of the proposed system and compared the results of them. There are some techniques has been used in this study for facial expression recognition such as Viola-Jones approaches to detect a face from images, Gabor Feature approach to extract features, and Principal Component Analysis (PCA) to select features and $k$-NN, SVM classifiers to recognize expressions from facial image.. The result showed that the SVM classifier has the best recognition rate in general than $k$-NN classifier. From these results, it can say that SVM classifier is more suitable for recognition of facial expression in a real-time system.
\end{abstract}

\section{General Terms}

Facial Expression Recognition, Pattern Recognition.

\section{Keywords}

Real-time System, Facial Expression Recognition, Classification, SVM, $k$-NN, Image Processing.

\section{INTRODUCTION}

Today, modern technology tried to exploit the wisdom of Allah in the creation of Human faces; where Faces are a unique feature of human being that can detect a great deal of information about age, health, personalities and feelings. Also, Human faces provide facial information as a clue for a better understanding the current state of user's mind. Accordingly, at recent days, Face recognition have attracted the attention and interesting the scientific community as well as the public.

Face recognition is one of the few biometric methods that involve the identification of human faces from a digital still image or a video sequence by comparing facial features extracted from the image and a facial database. It has various practical applications like surveillance system which is needed for effective communications and interactions among people.

Nowadays Human Computer Interaction (HCI) focused on development a natural human interaction with the computers, but it needs to have the communication skills of humans. One of these skills is the capacity to know status emotional of the person, facial expressions is one of the most expressive ways that displays emotions of humans.

Facial expressions is the facial changes in response to a person's intentions, or social communications, internal emotional states so it is one of the most robust channels of nonverbal communication that it is displayed by moving the muscles beneath the skin of the face. Thirty years ago, Paul Ekman identified the seven basic emotions through facial expressions (Anger, Disgust, Fear, Happiness, Nervous, Sadness and Surprise) [1].

An automatic facial expression recognition system (AFERS) is a computer system that aims to classify these changes or deformations into abstract classes automatically. It has many applications in areas like image understanding; psychological studies [2] [3] [4], and smarter human computer interfaces, elearning system [5] [6] [7] where behaviors of students can be studied through their facial as studies have proven that it contributes 55 percent to the effect of communicating a message [8]. Considering these applications, we find the facial expression recognition systems would be ineffective unless they are made to work in real-time.

Generally, the various phases involved in real-time facial expression recognition are face detection is the first phase; that aims to automatically locate a human face in an input image or image sequences. There are various approaches for face detection in real-time such as adaptive skin color [9], [10], Haar classifier [11], Adaboost [12] [13] and contour points [14] .Feature extraction is the second phase; that aims to locate specific features in a facial image such as points or contours by selecting features in order to decrease the number of features provided to the classification task. There are mainly three approaches for feature extraction - appearance based; this approach algorithms include Gabor wavelet [15], LBP [16], HOG [17], LDP [18], Gabor filter based texture information [19] and geometric based; this approach algorithms are AAM, Canny edge detection [10], active shape model (ASM) [20]. In addition to Hybrid features; that uses a geometric approach beside appearance-based approaches to extract features. Facial expression classification or feature matching is the final phase; it is the actual recognition process where the feature vector is obtained by the feature extraction. It is classified or matched to classes of facial expression images already enrolled in a database. Classification algorithms for facial expression recognition [21] are usually space-based methods like neural networks [22], support vector machines (SVM), $k$ - NearestNeighbor $(k-\mathrm{NN})$ [23] and AdaBoost method [24], etc. Time and space based methods are like hidden Markov model 
(HMM) [25], regression neural network, spatial and temporal motion energy templates method.

In this study, a real-time system for recognizing facial expression of the student during learning inside E-Learning environment was proposed. $k$-NN and SVM classifiers used to test efficiency system and compared the results of them.

\section{MATERIALS AND METHODS}

The following section clarify techniques used for facial expression recognition

\subsection{Face Detection}

The proposed system use adaptive version of Viola-Jones face detector that achieves real-time detection by executing two main steps: a) Haar-like features extraction and b) AdaBoost classifier; these steps represent Viola-Jones framework [26].

Haar-like features are rectangular features in a digital image, which are used in object detection. The integration of such features in a cascade generates a strong classifier out of the weak classifiers. Figure 1 shows some Haar-like features [27].

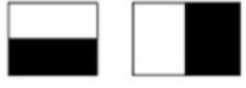

(a)

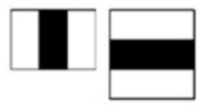

(b)

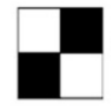

(c)
Figure 1: Common Haar features.

(a) Edge features. (b) Line features. (c) Four-rectangle features

For quick calculation of rectangular Haar-like features, integral images are computed Figure 2. Each element of the integral image contains the sum of all pixels located on the up-left region of the image. Using this, the sum of rectangular areas in the image at any position or scale is calculated. The integral image $I(x, y)$ is calculated efficiently in a single pass over the image using:

$$
I(x, y)=i(x, y)+I(x-1, y)+I(x, y-1)-I(x-1, y-1)
$$

In (1), $I(x, y)=\sum_{\substack{x^{\prime} \leq x \\ y^{\prime} \leq y}} i\left(x^{\prime}, y^{\prime}\right)$ and $i(x, y)$ is the intensity at $\operatorname{point}(x, y)$.

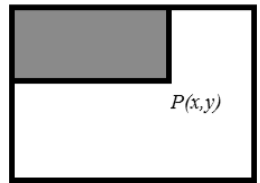

(a)

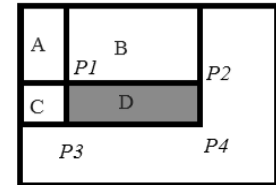

(b)
Figure 2: Integral image representation; (a)-The value of the integral image at point $(x, y)$ is the sum of all the pixels above and to the left. (b) The sum of pixels within $D$ can be computed as 4+1-(2+3).

AdaBoost robust classifier is applied (which is based on Rectangle Features) to decide whether the search window contains a face or not. Robust Classifier AdaBoost: linear structure of weak classifiers. $\mathrm{H}(\mathrm{x})=1$ if window $\mathrm{x}$ has a face and 0 otherwise.

$$
H(x)=\left\{\begin{array}{cc}
1 & \text { if } \sum_{t=1}^{T} \alpha_{t} h_{t}(x) \geq \emptyset \\
0 & \text { otherwise }
\end{array}\right.
$$

where $\alpha_{t}$ is Weights, $h_{t}$ is Weak Classifier and $(x) \geq \emptyset$ is Threshold.

$$
h_{j}(x)=\left\{\begin{array}{rr}
1 & \text { if } P_{j} f_{j}(x)<P_{j} \theta_{j} \\
0 & \text { otherwise }
\end{array}\right.
$$

where $f_{j}(x)$ is Rectangle Feature, $P_{j}$ is Sign and $\theta_{j}$ is Threshold.

\subsection{Feature Extraction and Selection}

The proposed system used Gabor Feature approach to extract features due to their powerful properties where it's optimal localization properties in each of the spatial and frequency domains and then select features using Principal Component Analysis (PCA).This method divides into three steps: Step1: generate a custom Gabor filter bank [28], Step2: Feature Extraction, Step3: Feature Selection using (PCA). A Gabor filter is a linear filter applied to images to extract features aligned at particular angles (orientations) and scale tuneable edge and line detectors. A Gabor filter can be represented by the following equation: [29]

$$
\begin{gathered}
\psi(x, y, \varpi, \theta)=\frac{1}{2 \pi \sigma^{2}} e^{-\left(\frac{x^{\prime 2}+y^{\prime} 2}{2 \sigma^{2}}\right)}\left[e^{i \varpi x^{\prime}}-e^{-\frac{\varpi^{2} \sigma^{2}}{2}}\right] \\
x^{\prime}=x \cos \theta+y \sin \theta, y^{\prime}=-x \sin \theta+y \cos \theta
\end{gathered}
$$

where $(x, y)$ is the pixel position in the spatial domain, $\varpi$ the radial center frequency, $\theta$ the orientation of Gabor filter, $\sigma$ the standard deviation of the round Gaussian function along the $\mathrm{x}$ and $y$-axes, and the second term of the Gabor filter, $e^{\wedge}\left(-\left(\varpi^{\wedge} 2 \sigma^{\wedge} 2\right) / 2\right)$ compensates for the DC value because the cosine component has nonzero mean while the sine component has zero mean.

Feature extraction is expressed by $i(x, y)$. Given a Gabor filter $g_{m n}(x, y)$ which is of $m$ th scale and $n$th orientation, the Gabor transform of the image is then defined as:

$$
W_{m n}(x, y)=\int i\left(x_{1}, y_{1}\right) \cdot g_{m n}\left(x-x_{1}, y-y_{1}\right) d x_{1} d y_{1}(5)
$$

This convolution process produces an equal sized output image for each single Gabor filter. Forty Gabor filters in five scales and eight orientations was used [30], as shown in Figure3.

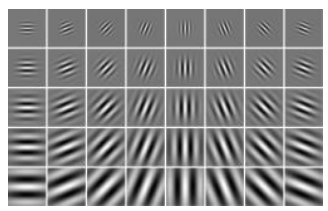

Figure3: Gabor wavelets in five scales and eight orientations

PCA was used to select features by reducing the dimension of data using the dependencies between the feature vectors and increase the speed of computation in real time. The steps involved are as follows [11]:

1. Each image is transformed into a vector of size $\mathrm{N}$ and placed into the set.

$$
S=\left\{\Gamma_{1}, \Gamma_{2}, \Gamma_{3}, \ldots, \Gamma_{\mathrm{M}}\right\}
$$

2. Mean feature vector is calculated using

$$
\psi=\frac{1}{\mathrm{M}} \sum_{i=1}^{\mathrm{M}} \Gamma_{i}
$$

3. Mean feature vector is subtracted from each feature vector $\Gamma_{\mathrm{i}}$

$$
\phi_{i}=\Gamma_{i}-\psi_{i}
$$

4. The covariance matrix $\mathrm{C}$ is estimated using the equation

$$
C=\frac{1}{\mathrm{M}} \sum_{i=1}^{\mathrm{M}} \phi_{i} \phi_{i}^{\mathrm{T}}=\mathrm{AA}^{\mathrm{T}}
$$


where $\mathrm{A}=\left[\phi_{1}, \phi_{2}, \ldots, \phi_{\mathrm{M}}\right]$ of $\quad$ dimension $h w \times \mathrm{M}$. Computing SVD of this large matrix is arithmetically difficult. Therefore, alternative method for finding eigen directions is applied. $\mathrm{A}^{\mathrm{T}}$ Ais computed instead of $\mathrm{AA}^{\mathrm{T}}$ as $\mathrm{M} \ll \mathrm{h}$, where the eigenvectors $e_{i}$ and eigenvalues $\lambda_{i}$ can be obtained by finding eigenvectors and eigen values of matrix $c=\mathrm{A}^{\mathrm{T}} \mathrm{A}$ (dimensions $M \times M$ ) [31] [32].

5. The eigenvectors $v_{i}$ of $A^{\mathrm{T}} A$ are calculated by

$$
\sigma_{i} u_{i}=A v_{i}
$$

hence the eigen-vectors $u_{i}$ of $A A^{\mathrm{T}}$ are obtained

6. Keep only $\mathrm{K}$ eigenvectors corresponding to the $\mathrm{K}$ largest Eigen values from each class (suppose $\left.\mathrm{U}=\left[\mathrm{u}_{1}, \mathrm{u}_{2}, \ldots, \mathrm{u}_{\mathrm{k}}\right]\right)$.

7. All the $\mathrm{K}$ eigenvectors are normalized.

\subsection{Classification of Facial Expression}

In real-time, the classification algorithm performance should be high in terms of both accuracy and efficiency. There are various approaches for classification in real-time domain for facial expression recognition. In this work, the proposed system is evaluated with two different classifiers: $k-\mathrm{NN}$ and SVM.

\subsubsection{Support Vector Machines (SVM) Classifier}

SVM [33] is a beneficial technique for data classification. Consider a training set of instance-label pairs $\left(x_{i}, y_{i}\right), i=$ $1, \ldots, 1$

where $x_{i} \in R^{n}$ and $y \in\{1,-1\}$. The SVM requires the solution of the following improvement problem:

$$
\min _{w, b, \xi} \frac{1}{2} w^{T} w+C \sum_{i=1}^{1} \xi_{i},
$$

subject to the constraints: $y_{i}\left(w^{T} \phi\left(x_{i}\right)+b\right) \geq 1-\xi_{i}, \xi_{i} \geq 0$ Training vectors are mapped into a higher dimensional space by the function $\emptyset$.

Furthermore, $K\left(x_{i}, x_{j}\right) \equiv \emptyset\left(x_{i}\right)^{T} \emptyset\left(x_{j}\right)$ is called the kernel function.

The choice of kernel function is also significant when designing SVM-based classifiers. There are two basic kernels that are usually used:

$$
\text { Linear function: } K\left(x_{i}, x_{j}\right)=x_{i}^{T} x_{j}
$$

Radial basis function (RBF):

$$
K\left(x_{i}, x_{j}\right)=\left(\gamma x_{i}^{T} x_{j}+r\right)^{d}, \gamma>0
$$

The prevailing approach for forming a multiclass SVM is to decrease the single multiclass problem into multiple binary classification problems [34]. There are two methods (i) one-versus-all that is applied by a winner-takes-all method, in which the classifier with the highest output function determines the class or (ii) one-versus-one that is applied by a max-wins voting method, in which every classifier determines the instance to one of the two classes, then the vote for the assigned classis increased by one vote, and finally the class with the most votes determines the instance classification. The one-versus-all approach was applied [35] to turn the binary SVM classifiers into a multi-label classifier.

\subsubsection{K-Nearest Neighbor (K-NN) Classifier}

In this classifier image is classified by a plurality vote of its neighbors. In $k$-NN classifier the Euclidean distance between each training image feature and the testing image feature is identified to form a distance matrix. The summation value of distance matrix is estimated and sorted in increasing order .The first $\mathrm{K}$ elements are chosen and majority class value is identified for classifying the image accurately [36][37].

$$
\text { Euclidean Distance }=\sqrt[2]{\sum_{\mathrm{i}=1}^{\mathrm{n}}\left(x_{i}-y_{i}\right)^{2}}
$$$$
\text { where } x=\left[x_{1}, x_{2}, x_{3}, \ldots, x_{n}\right] \text { and } y=\left[y_{1}, y_{2}, y_{3}, \ldots, y_{n}\right] \text { [38]. }
$$

\section{THE PORPOSED SYSTEM}

The goal of this study is to design a real-time system for facial expression recognition for students during learning inside elearning environment. Figure 4 shows the user interface that allows the student to select the lesson wants to learn and at the same time running webcam to capture images for the student for recognition the facial expression by proposed system. The overall system model in real-time for recognizing facial expression consists of four major phases: face acquisition, preprocessing of image face, feature extraction and facial expression classification. Figure 5 outlines the main phases of the proposed system.

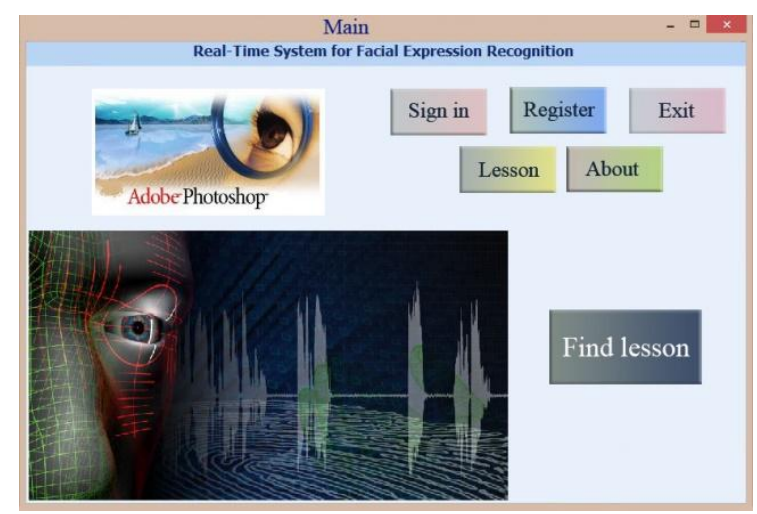

Figure4: The student's interface

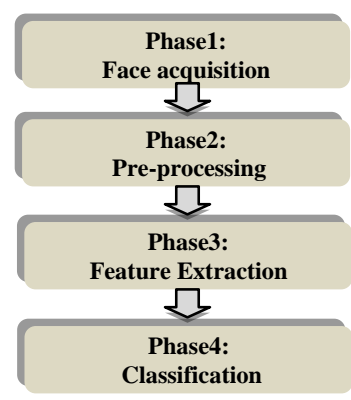

Figure 5: The overall of the real-time system phases.

The first phase is face acquisition aims to capture real-time images using webcam camera then detect or locate face regions from this capture images using Viola-Jones approach. Figure 6 illustrates of the original image and the image after detected face and cropping.

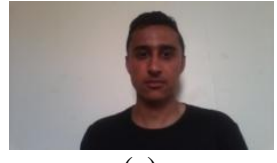

(a)

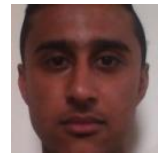

(b)
Figure6: (a) The original image, (b) the image after applying face detection and cropping

The second phase is pre-processing; it consists of many main operations, which applied on the face image to improve the quality of image and recognition rate. After detecting face from 
the image capture, the face image cropping is color; this system was dealing with images that contain Gray scale. So must convert the image to grayscale. Then resize the image by making the constant size of each image $(136,136)$ pixel. Image with uncontrolled lighting conditions contains non-uniform contrast, i.e. the distribution of gray levels / intensity is not alike. To make these levels equal or nearly equal we use histogram equalization technique [39]. Mathematically it is given as [40]

$$
h=\left(\frac{C D f(V)-C D f \min }{M X N-C D f \min }\right)(L-1)
$$

where given an $\mathrm{M} \times \mathrm{N}$ image, Cumulative Distribution Function $(C D f)$ at each Pixel Value $(\mathrm{V})$ is used to find pixels equalization value (h) over L Gray levels.

Also by default, images usually have Gaussian noise because of the difference of illumination. For this reason, pixel-based filtering techniques were used to de-noise it [41]. Low Pass Filter (LPF) was used to remove high-frequency information and keep low-frequency information only. The third phase is feature extraction where face image after pre-processing fed to a feature extraction. Gabor Feature approach was used to extract features and then reduce the dimensions to select features using (PCA). By using forty Gabor filters [42] [43], the feature vector dimension is $136 \times 136 \times 40=739,840$. The feature images are down sampled by a factor of four, which means that the output of this phase would be feature vector will have a size of $739,840 /(4 \times 4)=46,240$. These vectors are then normalized to zero mean and unit variance. To further minimize the size of the feature vectors using (PCA)

The fourth phase is classification of facial expression which aims to recognize and classify expressions based on the extracted features. In this work, the proposed system is evaluated with two different classifiers: $k$-NN and SVM. The one vs. all approach was applied to turn the binary SVM classifiers into a multi-label classifier. For the experiments, the LibSVM library [44] is used for applying nonlinear SVM on the proposed system during training and in testing phase.

The whole procedure is carried out in two phases i.e. training phase and testing phase. The flowcharts for the training phase and testing phase are given in (Figure7).

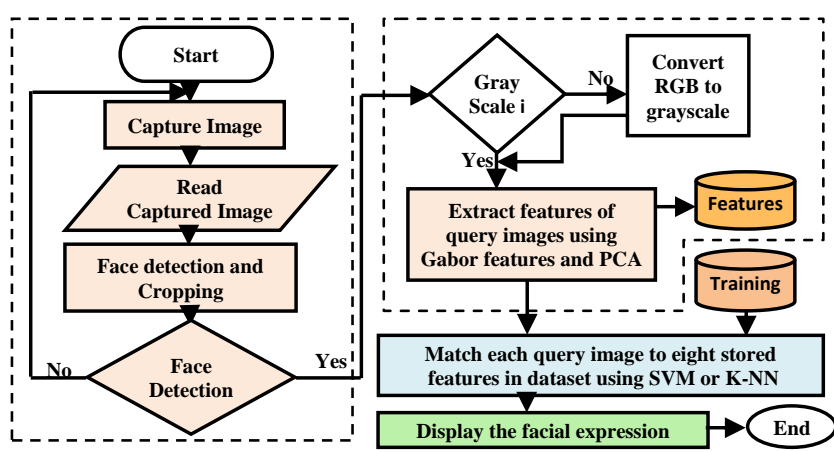

Figure7: The proposed system phases.

\section{EXPERMIENT AND DISCUSSION}

The proposed method was implemented in MATLAB 2014b. The system was run in an Intel core i3, 2GB of RAM and use 64-bit "Windows 7".

\subsection{Dataset Preparation}

This dataset prepared by capture images for different facial expression for 30 students using webcam in front of the student. where each student has 16 images that represent different expressions ('happiness', 'surprise', 'anger', 'disgust', 'sad', 'nervous' and 'fears') plus neutral expression for this student.

Figure 8 shows the example of a dataset for some student.

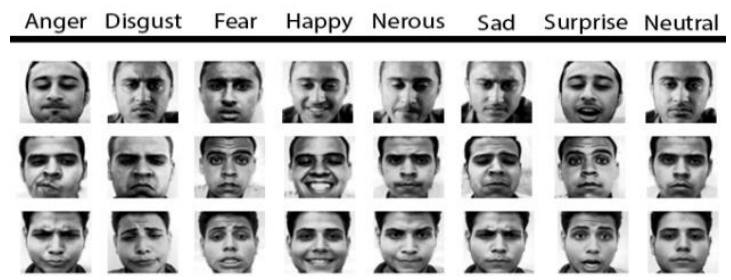

Figure 8: Examples of training dataset for some student.

\subsection{Experiment}

The proposed system test inside asynchronous e-learning environment where student after choose lesson that want to learn and click to start learning video at the same time webcam running to capture real-time facial expression images for student during learning, these images capture every 20 seconds until finishing 10 minutes for video. This means, the total number of test images, $(30 \times 30=900)$. Figure 9 shows example of facial expression images for some student captured during learning (testing dataset).

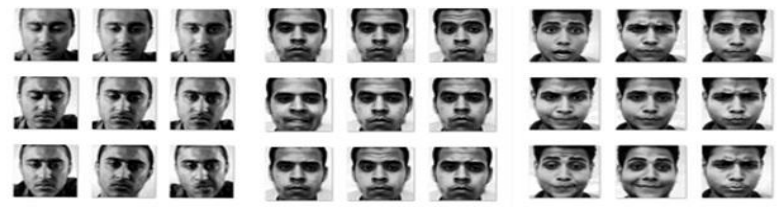

Figure9: Examples of facial expression images for some student captured during learning (testing dataset).

\subsection{Results and Discussion}

\subsubsection{Using $k$-NN classifier}

In this experiment use $k$-NN classification to measure the proposed system facial expressions recognition rate for student during learning inside e-learning environment. In this classification 650 images have true expression class and 250 images have false expression class. This means, the average of image recognition rate of proposed system using $k$-NN classification is $72 \%$ from all images of facial expressions (900 images) as shown in table1. In this Table each student's emotion is detached in two rows that intersect with the recognized emotions by the proposed system. The disgust and fear expressions is the highest recognition rate $(100 \%)$ and the neutral expression is the lowest recognition rate $(58 \%)$ for the proposed system.

Table 1: Recognized Facial Expression by the proposed system using $k$-NN classifier

\begin{tabular}{cccccccccc}
\hline $\begin{array}{c}\text { Out } \\
\text { In }\end{array}$ & An & Di & Fe & Ha & Ne & Sad & Sur & Na & $\begin{array}{c}\text { RR } \\
(\%)\end{array}$ \\
\hline An & 58 & 0 & 0 & 3 & 1 & 5 & 0 & 31 & 85 \\
Di & 0 & 20 & 0 & 0 & 1 & 1 & 0 & 15 & 100 \\
Fe & 0 & 0 & 6 & 0 & 0 & 0 & 1 & 2 & 100 \\
Ha & 1 & 0 & 0 & 83 & 1 & 0 & 0 & 42 & 91 \\
Ne & 3 & 0 & 0 & 0 & 77 & 2 & 1 & 36 & 85 \\
Sad & 2 & 0 & 0 & 0 & 3 & 79 & 1 & 53 & 88 \\
Sur & 1 & 0 & 0 & 3 & 1 & 1 & 50 & 24 & 93 \\
Na & 3 & 0 & 0 & 2 & 7 & 2 & 1 & 277 & 58 \\
\hline Total & 68 & 20 & 6 & 91 & 91 & 90 & 54 & 480 & \\
\hline
\end{tabular}

* An: angry, Di: disgust, Fe: fear, Ha: happy, Ne: nervous, Sad: sad, Sur: surprised, Na: neutral, RR: recognition rate 
The rows from Table 1 show that all 8basic emotions have several distributions for being confused as of the other emotions. In other words, they have various discrimination rates. The emotion that shows better discrimination from other emotions is disgust and fear, as disgust has a high score $100 \%$ and is not confused with angry, fear, happy and surprised. Also, as fear has a high score $100 \%$ and is not confused with angry, disgusted, happy, nervous and sad.

\subsubsection{Using SVM classifier}

In this experiment use SVM classification to measure the proposed system facial expressions recognition rate for student during learning inside e-learning environment. In this classification 822 images have true expression class and 78 images have false expression class. This means, the average of image recognition rate of proposed system using SVM classification is $91 \%$ from all images of facial expressions (900 images) as shown in table 2 . In this table, each student's emotion is detached in two rows that intersect with the recognized emotions by the proposed system. The fear expression is the highest recognition rate $(100 \%)$ and the nervous expression is the lowest recognition rate (79\%) for the proposed system.

Table 2: Recognized Facial Expression by the proposed system using SVM classifier

\begin{tabular}{cccccccccc}
\multicolumn{10}{c}{ System using SVM classifier } \\
$\begin{array}{c}\text { Out } \\
\text { In }\end{array}$ & An & Di & Fe & Ha & Ne & Sad & Sur & Na & $\begin{array}{c}\text { RR } \\
(\%)\end{array}$ \\
\hline An & 56 & 0 & 0 & 0 & 0 & 0 & 0 & 3 & 82 \\
Di & 0 & 18 & 0 & 0 & 0 & 0 & 0 & 0 & 90 \\
Fe & 0 & 0 & 6 & 0 & 0 & 0 & 0 & 0 & 100 \\
Ha & 0 & 0 & 0 & 78 & 0 & 0 & 0 & 0 & 86 \\
Ne & 1 & 0 & 0 & 0 & 72 & 1 & 0 & 2 & 79 \\
Sad & 0 & 0 & 0 & 0 & 0 & 72 & 0 & 4 & 80 \\
Sur & 1 & 0 & 0 & 0 & 0 & 0 & 49 & 0 & 91 \\
Na & 10 & 2 & 0 & 13 & 19 & 17 & 5 & 471 & 98 \\
\hline Total & 68 & 20 & 6 & 91 & 91 & 90 & 54 & 480 & \\
\hline
\end{tabular}

* An: angry, Di: disgust, Fe: fear, Ha: happy, Ne: nervous, Sad: sad, Sur: surprised, Na: neutral, RR: recognition rate

The rows from Table 2 show that all 8basic emotions have several distributions for being confused as of the other emotions. In other words, they have various discrimination rates. The emotion that shows better discrimination from other emotions is fear, as fear has a high score $100 \%$ and is not confused with angry, disgusted, happy, nervous, sad and neutral.

\subsubsection{Comparison between the result of $k-N N$ and SVM}

The facial expression recognition performances using $k$-NN and SVM classifier are displayed in Table 3 and Figure 10. From this figure, we can find that the average facial expression recognition rate of SVM Classifier is better than $k-\mathrm{NN}$.

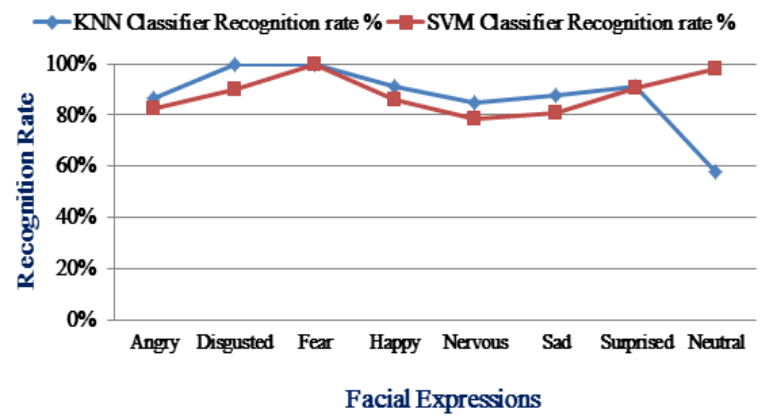

Figure10: Comparison between $k$-NN and SVM classifiers.
Table 3: The comparison of facial expressions recognition performances

\begin{tabular}{lcc}
\hline \multicolumn{1}{c}{$\begin{array}{c}\text { Facial } \\
\text { Expressions }\end{array}$} & $\begin{array}{c}\boldsymbol{k} \text {-NN Classifier } \\
\text { Recognition rate }\end{array}$ & $\begin{array}{c}\text { SVM Classifier } \\
\text { Recognition rate \% }\end{array}$ \\
\hline Angry & $85 \%$ & $82 \%$ \\
Disgust & $100 \%$ & $90 \%$ \\
Fear & $100 \%$ & $100 \%$ \\
Happy & $91 \%$ & $86 \%$ \\
Nervous & $85 \%$ & $79 \%$ \\
Sad & $88 \%$ & $80 \%$ \\
Surprised & $93 \%$ & $91 \%$ \\
Neutral & $58 \%$ & $98 \%$ \\
Average & $\mathbf{8 7 \%}$ & $\mathbf{8 8 \%}$ \\
\hline
\end{tabular}

From Table 3, it can conclude that:

1. Although the $k-\mathrm{NN}$ is known as one of the best classifiers, it showed the bad performance in recognition rate for the images of a neutral expression.

2. The $k$-NN classifier shows the good recognition performance in all expression, excepting in the neutral image facial expressions due to recognition rate for neutral expression is $58 \%$ from the total image so that the $k$-NN classifier performance is the lowest recognition rate.

3. The SVM classifier performance is the highest recognition rate for facial expressions and image recognition.

4. If normal image deleted, the average recognition rate for $k$-NN will improve, but the proposed system is real life so do not be separated normal image of student which indicates that it doesn't issue any negative or positive interaction with the system.

5. By comparing these results with the results of some recent papers on the real-time system, note that in [45] used SVM to classify 6 facial expressions and the recognition rate was about $93 \%$ in a real-world environment. Also, in [46] study evaluate the performance of three different classifiers using Multilayer perceptrons (MLPS), SVM and AdaBoost algorithms, the accuracy rate of facial expression recognition system achieved more than $90 \%$ for expressions appearing in an image sequence.

\section{CONCLUSION}

This paper has presented a real-time facial expression recognition system that aims to recognize the 8 basic facial expressions of students' anger, disgust, fear, happy, nervous, sad, surprise, and natural during learning time inside E-learning environment. $k$-NN and SVM classifiers used to test efficiency system and compared the results of them. The experimental results showed that SVM had the best recognition rate in neutral facial expiration for all students than $k$-NN. In future, the proposed system will be extended to cover more students and process the illumination problem as well as more classifiers will be performed to improve the proposed system accuracy.

\section{ACKNOWLEDGMENTS}

The authors thank all students for their collaboration in the experiments. 


\section{REFERENCES}

[1] Ekman, P., \& Friesen, W. V. (1978). The facial action coding system: a technique for the measurement of facial movement. Consulting Psychologists Press, Palo Alto.

[2] Alazrai, R., \& Lee, C. G. (2012, May). Real-time emotion identification for socially intelligent robots. In Robotics and Automation (ICRA), 2012 IEEE International Conference on (pp. 4106-4111). IEEE.

[3] Lau, B. T. (2010). Portable real time emotion detection system for the disabled. Expert Systems with Applications, 37(9), 6561-6566.

[4] Lu, X. (2003). Image analysis for face recognition. personal notes, May, 5.

[5] Grafsgaard, J., Wiggins, J. B., Boyer, K. E., Wiebe, E. N., \& Lester, J. (2013, July). Automatically recognizing facial expression: Predicting engagement and frustration. In Educational Data Mining 2013.

[6] Littlewort, G., Whitehill, J., Wu, T., Fasel, I., Frank, M., Movellan, J., \& Bartlett, M. (2011, March). The computer expression recognition toolbox (CERT). In Automatic Face \& Gesture Recognition and Workshops (FG 2011), 2011 IEEE International Conference on (pp. 298-305). IEEE.

[7] Sathik, M., \& Jonathan, S. G. (2013). Effect of facia expressions on student's comprehension recognition in virtual educational environments. Springer Plus, 2(1), 1.

[8] Loh, M. P., Wong, Y. P., \& Wong, C. O. (2005, July). Facial expression analysis in e-learning systems-the problems and feasibility. In Fifth IEEE International Conference on Advanced Learning Technologies (ICALT'05) (pp. 442-446). IEEE.

[9] Peng, Z. Y., Wen, Z. Q., \& Zhou, Y. (2009, January). Application of mean shift algorithm in real-time facial expression recognition. In Computer Network and Multimedia Technology, 2009. CNMT 2009. International Symposium on (pp. 1-4). IEEE.

[10] Zhao-yi, P., Yan-hui, Z., \& Yu, Z. (2010, April). Realtime facial expression recognition based on adaptive canny operator edge detection. In Multimedia and Information Technology (MMIT), 2010 Second International Conference on (Vol. 2, pp. 154-157). IEEE.

[11] N. Bajaj and A. Routray (2013): "Dynamic Model of Facial Expression Recognition based on Eigen-face Approach", Proceedings of Green Energy and Systems Conference 2013, November 25, Long Beach, CA, USA

[12] Srivastava, S. (2012, January). Real Time Facia Expression Recognition. In International Conference on Computer Science and Information Technology (pp. 124133). Springer Berlin Heidelberg.

[13] Wang, Y., Ai, H., Wu, B., \& Huang, C. (2004, August) Real time facial expression recognition with adaboost. In Pattern Recognition, 2004. ICPR 2004. Proceedings of the 17th International Conference on (Vol. 3, pp. 926-929). IEEE.

[14] Geetha, A., Ramalingam, V., Palanivel, S., \& Palaniappan, B. (2009). Facial expression recognition-A real time approach. Expert Systems with Applications, 36(1), 303-308.
[15] Adeshina, A.M., Lau, S.-H., Loo, C.-K. (2009). Real-time FERs: a review. Innovative Technologies Intelligent Systems and Industrial Applications, pp. 375-378

[16] Happy, S. L., George, A., \& Routray, A. (2012, December). A real time facial expression classification system using Local Binary Patterns. In Intelligent Human Computer Interaction (IHCI), 2012 4th International Conference on (pp. 1-5). IEEE.

[17] Dalal, N., \& Triggs, B. (2005, June). Histograms of oriented gradients for human detection. In 2005 IEEE Computer Society Conference on Computer Vision and Pattern Recognition (CVPR'05) (Vol. 1, pp. 886-893). IEEE.

[18] Jabid, T., Kabir, M. H., \& Chae, O. (2010, September) Facial expression recognition using local directional pattern (LDP). In 2010 IEEE International Conference on Image Processing (pp. 1605-1608). IEEE.

[19] Verma, D., Saini, L.K., Joshi, K. (2013). Performance analysis of feature extraction technique for facia expression recognition. Int. J. Adv. Comput. Technol. (IJACT), 2(4), pp. 16-20

[20] Ahn, B., Han, Y., \& Kweon, I. S. (2012, November) Real-time facial landmarks tracking using active shape model and $\mathrm{lk}$ optical flow. In Ubiquitous Robots and Ambient Intelligence (URAI), 2012 9th International Conference on (pp. 541-543). IEEE.

[21] Wu, T., Fu, S., \& Yang, G. (2012, July). Survey of the facial expression recognition research. In International Conference on Brain Inspired Cognitive Systems (pp. 392-402). Springer Berlin Heidelberg.

[22] Lee, H. C., Wu, C. Y., \& Lin, T. M. (2013). Facial Expression Recognition Using Image Processing Techniques and Neural Networks. In Advances in Intelligent Systems and Applications-Volume 2 (pp. 259 267). Springer Berlin Heidelberg.

[23] Song, Y., Huang, J., Zhou, D., Zha, H., \& Giles, C. L. (2007, September). Iknn: Informative k-nearest neighbor pattern classification. In European Conference on Principles of Data Mining and Knowledge Discovery (pp. 248-264). Springer Berlin Heidelberg.

[24] Wang, Y., Ai, H., Wu, B., \& Huang, C. (2004, August). Real time facial expression recognition with adaboost. In Pattern Recognition, 2004. ICPR 2004. Proceedings of the 17th International Conference on (Vol. 3, pp. 926-929). IEEE.

[25] Schmidt, M., Schels, M., \& Schwenker, F. (2010, April) A hidden markov model based approach for facial expression recognition in image sequences. In IAPR Workshop on Artificial Neural Networks in Pattern Recognition (pp. 149-160). Springer Berlin Heidelberg.

[26] Viola, P., \& Jones, M. (2001). Robust real-time object detection. International Journal of Computer Vision, 4

[27] Wilson, P. I., \& Fernandez, J. (2006). Facial feature detection using Haar classifiers. Journal of Computing Sciences in Colleges, 21(4), 127-133.

[28] Lee, T. S. (1996). Image representation using 2D Gabor wavelets. IEEE Transactions on pattern analysis and machine intelligence, 18(10), 959-971. 
[29] Deng, H. B., Jin, L. W., Zhen, L. X., \& Huang, J. C. (2005). A new facial expression recognition method based on local gabor filter bank and pca plus lda. International Journal of Information Technology, 11(11), 86-96.

[30] Haghighat, M., Zonouz, S., \& Abdel-Mottaleb, M. (2015). CloudID: trustworthy cloud-based and cross-enterprise biometric identification. Expert Systems with Applications, 42(21), 7905-7916.

[31] Turk, M. A., \& Pentland, A. P. (1991, June). Face recognition using eigen faces. In Computer Vision and Pattern Recognition, 1991. Proceedings CVPR'91., IEEE Computer Society Conference on (pp. 586-591). IEEE.

[32] Vyas, A., \& Tokas, R. Fast Face Recognition Using Eigen Faces.

[33] Chen, L., Zhou, C., \& Shen, L. (2012). Facial Expression Recognition Based on SVM in E-learning. IERI Procedia, $2,781-787$.

[34] Duan, K. B., \& Keerthi, S. S. (2005, June). Which is the best multiclass SVM method? An empirical study. In International Workshop on Multiple Classifier Systems (pp. 278-285). Springer Berlin Heidelberg.

[35] Jottrand, M. (2005). Support Vector Machines for Classification applied to Facial Expression Analysis and Remote Sensing.

[36] Maheswari, K. S. \& Babu, C. H. (2015). A Color Face Recognition Using PCA and KNN Classifier, international journal \& magazine of engineering technology, management and research, vol2, issues: 9, pp.1110-1116.

[37] Ewees, A. A., Eisa, M., \& Refaat, M. M. (2014) Comparison of cosine similarity and k-NN for automated essays scoring. Cognitive processing, 3(12).

[38] Suja, P., Tripathi, S., \& Deepthy, J. (2014). Emotion recognition from facial expressions using frequency domain techniques. In Advances in signal processing and intelligent recognition systems (pp. 299-310). Springer International Publishing.

[39] Zhang, B., Shan, S., Chen, X., \& Gao, W. (2007). Histogram of Gabor phase patterns (HGPP): a novel object representation approach for face recognition. IEEE Transactions on Image Processing, 16(1), 57-68.

[40] Abuqaaud, K. A. (2013). Face Recognition in Uncontrolled Indoor Environment (Doctoral dissertation, American University of Sharjah).

[41] Dong-liang, P., \& An-ke, X. (2005, October). Degraded image enhancement with applications in robot vision. In 2005 IEEE International Conference on Systems, Man and Cybernetics (Vol. 2, pp. 1837-1842). IEEE

[42] Liu, C., \& Wechsler, H. (2002). Gabor feature based classification using the enhanced fisher linear discriminate model for face recognition. IEEE Transactions on Image processing, 11(4), 467-476.

[43] Shen, L., Bai, L., \& Fairhurst, M. (2007). Gabor wavelets and general discriminant analysis for face identification and verification. Image and Vision Computing, 25(5), 553-563.

[44] Chang, C. C., \& Lin, C. J. (2001). LIBSVM: A library for support vector machines, Software available at $\mathrm{h} t \mathrm{ttp}$. WWW. CSIE. NTU. EDU. TW/ CJLIN/ PAPERS/ LIBSVM.

[45] Hsieh, C. C., Hsih, M. H., Jiang, M. K., Cheng, Y. M., \& Liang, E. H. (2015). Effective semantic features for facial expressions recognition using SVM. Multimedia Tools and Applications, 1-20.

[46] Fahn, C. S., Wu, M. H., \& Kao, C. Y. (2009, October). Real-time facial expression recognition in image sequences using an AdaBoost-based multi-classifier. In Proceedings: Asia-Pacific Signal and Information Processing Association, 2009 Annual Summit and Conference (pp. 8-17). 